\title{
Postoperative analgesia for cesarean deliveries during CSE: technical maintenance compared
}

Marco Aversano ${ }^{1},{\text { Claudia } \text { Claroni }^{1} \text {,Elisabetta Soluri }}^{2}$, Cristina Todde ${ }^{1}$, Maria Grazia Frigo ${ }^{1}$

1. Department of Obstetric Anesthesia, S.G. Calibita Fatebenefratelli Isola Tiberina Hospital, Rome, Italy

2 Department of Anesthesiology and Intensive Care, II Faculty of Medicine and Psychology, "Sapienza" University, Rome.

Background and Goal of Study: The post operative pain after caesarean is a moderate/intense pain, which, if not treated properly, it can give negative effects not only for the mother but also for the new born: it could prevail survival instinct about their "pain disease" rather than instinctive offspring caregiving. The aim of our study was to compare two techniques of maintaining post operative analgesia in caesarean section

Materials and Methods: It was carried out an observational study in which two groups of parturient submitted to caesarean deliveries have received a combined spinal-epidural anaesthesia; then, were divided into two groups. The study group received an analgesic pump in PIEB mode (programmed intermittent epidural bolus) and the control group received a CEI pump mode (continuous epidural infusion ) connected to the epidural catheter. In both cases, the patients were instructed to selfadminister additional boluses of medication in case of pain. The degree of analgesia satisfaction and the occurrence of side effects (such as vomiting, itching, numbness, motor block) were assessed by a questionnaire. Data about self-administered boluses numbers registered by the analgesic pumps were analysed.

Results and Discussion: 120 patients undergoing caesarean section were enrolled in this study, 60 in PIEB group and 60 in the CEI group. Questionnaires have revealed that the average reported pain in the CEI group evaluated according to NRS scale, is 6.3 compared with an average of 2.7 of PIEB group ( $p>0.001$ ). The consumption in the two groups is expressed in Figure 1.

Conclusions: In our study the PIEB technique is better for pain control during 24 hours after caesarean section: in fact there was a lower consumption of the drug compared to the CEI technique and it also revealed a greater maternal satisfaction regarding pain relief .

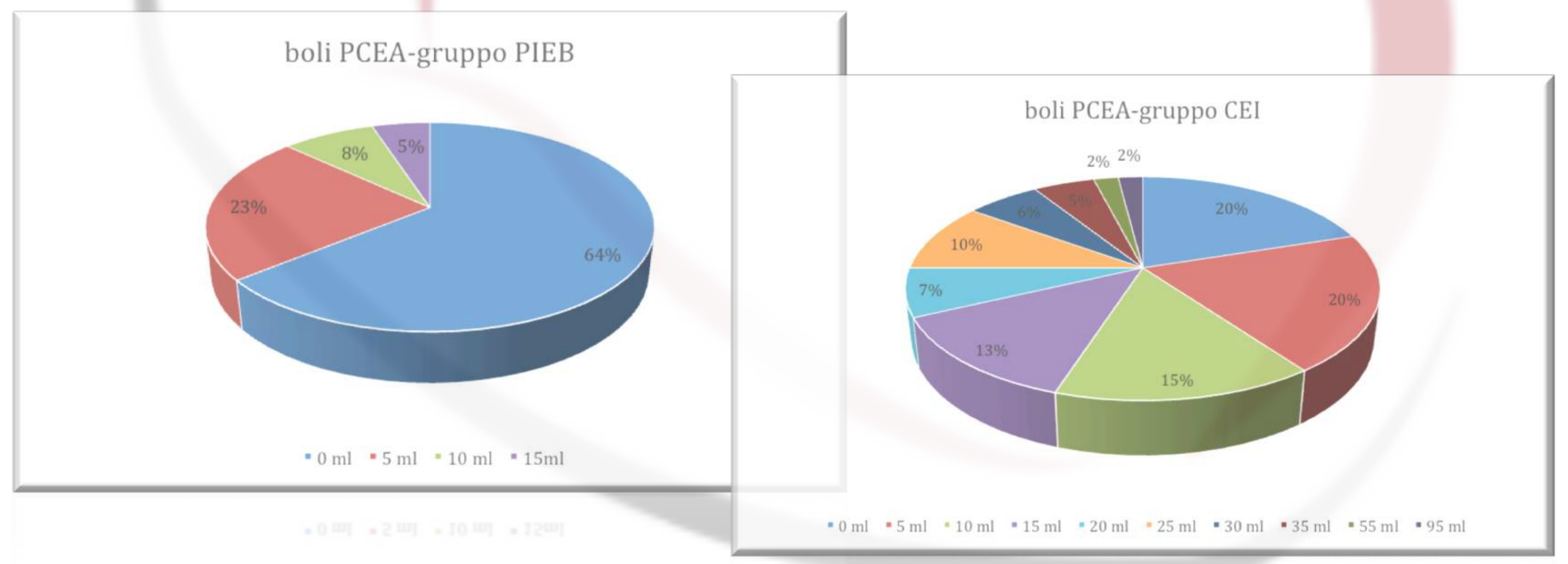

